\title{
Lipids in bronchoalveolar lavage fluid from patients with sarcoidosis
}

\author{
R B LOW, G S DAVIS, DIANNE Y BELL, M S GIANCOLA, PAMELA M VACEK \\ From the Department of Physiology and Biophysics, the Department of Medicine, and the Department of \\ Medical Biostatistics, University of Vermont, Burlington; and the Department of Pediatrics, Duke University, \\ Durham, USA
}

\begin{abstract}
The recovery of protein and two specific surfactant lipids, phosphatidylcholine an $\Phi^{\$}$ phosphatidylglycerol, from bronchoalveolar lavage fluid is altered in chronic and acute non? granulomatous interstitial lung disease. This study set out to determine whether the same is true for patients with sarcoidosis. The median value for recovery of protein from lavage fluid was significantly higher in 21 patients with sarcoidosis than in 19 normal subjects $(18 v 11 \mathrm{mg})$, while the median value for phospholipid recovery was significantly lower $\left(\begin{array}{lll}4 & v & 1.7 \\ \mathrm{mg}\end{array}\right)$. There were no changes in the proportions of phosphatidylcholine and phosphatidylglycerol. In addition, significantly less of the neutral lipid, cholesterol, was recovered $(3.2 v 1.5 \mathrm{mg})$. The combined values of three biochemicapo measurements, non-phospholipid polar lipid, non-polar lipid, and protein, correctly classified all 40 subjects in our series; in a further group of nine normal subjects and 11 patients with sarcoidosis ie allowed all but one normal subject to be classified correctly. These results are discussed in terms of alterations in epithelial cell function in interstitial disease.
\end{abstract}

Diffuse interstitial pulmonary disease is manifested by a range of abnormalities of the interstitium, which ultimately affect lung function with debilitating consequences. Analysis of cells and proteins recovered by bronchoalveolar lavage has proved useful in understanding the nature of the abnormalities that occur and in formulating critical questions regarding pathogenesis. ${ }^{1-4}$ Much less information is available on changes in lipid constituents of the alveolus, particularly those that relate to the function of pulmonary surfactant.

Lipids generally are classified according to their chemical and physical properties rather than on a functional basis. Two major lipid groups are the uncharged neutral lipids, which include cholesterol and triglycerides, and charged or polar lipids, which include phospholipids. Phospholipids, because of the distribution of charge on their surface, are key components of cellular membranes. Certain phospholipids aIso possess the unusual ability to lower surface

Address for reprint requests: Dr Robert B Low, Department of Physiology and Biophysics, Given E-211, University of Vermont, Burlington, Vermont 05405, USA.

Accepted I July 1987 tension at air-water interfaces. Thus they are key components of pulmonary surfactant, responsible fo $\vec{E}$ reducing alveolar surface tension. Two specific phos pholipids, phosphatidylcholine and phosphatidyl glycerol, are major determinants of surfactant activity. ${ }^{5}$ Other lipids, including non-polar lipids suclo as cholesterol, are also of interest because they may be expected to interdigitate with and thereby alter the surface active properties of surfactant.

Specific changes in the amount and proportion of phosphatidylcholine and phosphatidylglycerot recovered from bronchoalveolar lavage fluid have been reported in non-granulomatous interstitial lung disease. ${ }^{67}$ Studies of various animal models have led to the same conclusion and suggest that such measure $\widetilde{N}$ ments might be useful in understanding the alteration in lung function that occur. ${ }^{8-11}$ The purpose of the present study was to determine the degree to whicb similar changes also occur in a granulomatous disease sarcoidosis. A preliminary report of these findings has appeared elsewhere. ${ }^{?}$

\section{Methods}

PATIENTS

Twenty one patients with sarcoidosis underwent 
detailed evaluation. This included routine medical history and physical examination; detailed occupational and environmental history; conventional and magnification chest radiographs; 12 lead electrocardiogram; and a panel of blood, urine, and skin tests. Pulmonary function was assessed by measurements of static and dynamic lung volumes, gas exchange at rest and during treadmill exercise, and single breath carbon monoxide diffusing capacity (transfer factor). A diagnosis of sarcoidosis was established in all patients by histological examination of samples obtained by transbronchial biopsy or mediastinoscopy. The chest radiographs were typed according to the method of Sharma. ${ }^{12}$ Nineteen healthy volunteers were studied as a control group.

\section{BRONCHOALVEOLAR LAVAGE}

Patients underwent lavage during bronchoscopy for transbronchial lung biopsy or at the same session as mediastinoscopy. Pulmonary lavage was performed in normal volunteers by out patient transnasal fibreoptic bronchoscopy. ${ }^{13}$ Lavage was performed by the instillation and immediate withdrawal of four $60 \mathrm{ml}$ boluses of sterile $\mathbf{0 . 9 \%}$ saline solution (Baxter Laboratories) through the channel of a $5.2 \mathrm{~mm}$ fibreoptic bronchoscope (Olympus Corporation), the tip of which had been gently wedged in a subsegmental bronchus of the right middle lobe. The lavage procedure was terminated if a residual volume of $100 \mathrm{ml}$ saline remained after a withdrawal step. All normal subjects and 16 of the patients had a complete lavage procedure $(4 \times$ $60 \mathrm{ml}$ ).

All the lavage fluid recovered from each subject was pooled and centrifuged at $400 \mathrm{~g}$ for 15 minutes at $25^{\circ} \mathrm{C}$. The cell free supernatants were immediately frozen at $-30^{\circ} \mathrm{C}$. The cell pellet was resuspended and a total cell count was determined with a haemocytometer. Viability was $90 \%$ or greater as determined by trypan blue dye exclusion. A portion of this cell preparation was spread on glass slides manually or by cytocentrifuge (Shandon), air dried, and stained by the May-Grünwald-Giesma technique. The percentages of alveolar macrophages, polymorphonuclear leucocytes, and lymphocytes were determined by differential cell counting of at least 300 cells per specimen.

\section{BIOCHEMICAL ANALYSES}

Portions of frozen lavage fluid were thawed at $4^{\circ} \mathrm{C}$ before being analysed directly for protein as described previously. ${ }^{13}$ Total lipid extracts were separated into non-polar and polar lipid fractions by silicic acid chromatography. Firstly, non-polar lipids were eluted with chloroform followed by methanol to elute polar lipids. ${ }^{13}$ Individual non-polar lipids were separated by one dimensional silica gel thin layer chromatography before quantitation by spectrophotometry of mala- chite green stained individual lipids, according to the methods of Freeman and West ${ }^{14}$ is as modified by Wuthier, ${ }^{16}$ except that the first solvent was hexane:2butanone:benzene:diethyl ether:acetic acid: ethanol (36:4:32:28:1:1(v/v)) and the second was hexane:diethyl ether $(94: 6(v / v))$. Polar lipids were separated by two dimensional paper chromatography before quantitation of each spot by phosphorus analysis, as previously described. ${ }^{13}$ The total lipid concentration was calculated as the sum of the concentrations of non-polar plus polar lipids. The phospholipid concentration was calculated as the lipid phosphorus concentration $\times 25 .^{14}$ Non-phospholipid polar lipid was calculated as the difference between polar lipid and phospholipid and expressed as a percentage of phospholipid. The percentage of phospholipid that was saturated was determined by the osmium methods of Mason et al. ${ }^{17}$ Control experiments to determine the accuracy of our lipid analyses were performed as before ${ }^{13}$ and included saline controls for which there was no recovery of lipid, quantitative recovery of lipid standards by themselves or added to lavage samples, and accurate assessment of the degree of phospholipid saturation, also determined with standards.

\section{STATISTICAL ANALYSIS}

Non-parametric tests were used because the data were non-normal. Mann-Whitney tests were used for comparison of median values between volunteers and patients with sarcoidosis, two sided comparisons being made in all cases. ${ }^{18}$ Comparisons between radiographic type used the Kruskal-Wallis test for median values and multiple comparison procedures to adjust significance levels when simultaneous tests were made. ${ }^{19} \mathrm{~A}$ log rank procedure for trend was used to determine whether a particular value increased or decreased when groups of patients were ordered with respect to radiographic type in the order type I, type II, type III. ${ }^{19}$

In addition to these univariate analyses, stepwise discriminant analysis was used to examine the ability of groups of variables to distinguish collectively between patients and normal subjects. ${ }^{20}$ This procedure identifies the linear combination of variables showing the greatest difference between the two groups. The magnitude of the standardised coefficients associated with each variable in the linear function indicates their relative abilities to distinguish the two groups of subjects. The signs of the coefficients reveal the pattern of change that characterise patients with sarcoidosis. In this analysis we excluded one patient whose lavage protein concentration was more than $2000 \mathrm{mg} / \mathrm{ml}$, because the stepwise discriminant analysis procedure is based on the assumption that the data are from a normally distributed population. 
Although the analysis is very robust with deviations from normality, this protein value was so large that it would have excessively distorted the estimated function.

\section{Results}

\section{CHARACTERISTICS OF THE STUDY GROUPS}

The seven male and 14 female patients with sarcoidosis ranged in age from 23 to 64 (median 34) years and 14 were non-smokers. Six of the patients had reduced vital capacity (less than $80 \%$ predicted), five had a resting oxygen tension $\left(\mathrm{Po}_{2}\right)$ below $80 \mathrm{~mm} \mathrm{Hg}$ $(10.7 \mathrm{kPa})$, and all but three had alveolar-arterial oxygen gradients $\left(\mathrm{PA}-\mathrm{aO}_{2}\right)$ above $15 \mathrm{~mm} \mathrm{Hg}(2 \mathrm{kPa})$ during exercise. All patients had an abnormal chest radiograph, seven being classified as type I (bilateral hilar lymphadenopathy), seven showing type II changes (bilateral hilar lymphadenopathy plus pulmonary infiltrates), and seven showing type III disease (pulmonary infiltrates alone). None of the patients was receiving corticosteroids at the time of evaluation and lavage.

The healthy volunteer group consisted of seven merk and 12 women, ranging in age from 20 to 42 years Eleven of the 19 subjects were smokers.

LAVAGE ANALYSES: GENERAL FEATURES

The recovery of fluid and cells from the patients and normal subjects gave results similar to the finding fw reported by others. The volume recovered from patients with sarcoidosis was not significantly different from control values. The total number of cells ret covered varied widely between individuals, smoking habit, volume of fluid, and presence of disease being the predominant determining factors. Specimens fromp smokers yielded nearly three times the number of cells obtained from non-smokers. The median value for percentage of lymphocytes was increased in the sarcoidosis patients. The lavage fluids from one sarcoidosis patient contained a high percentage of polymorphonuclear leucocytes.

Protein and lipids recovered in bronchoalveolar lavage fluid

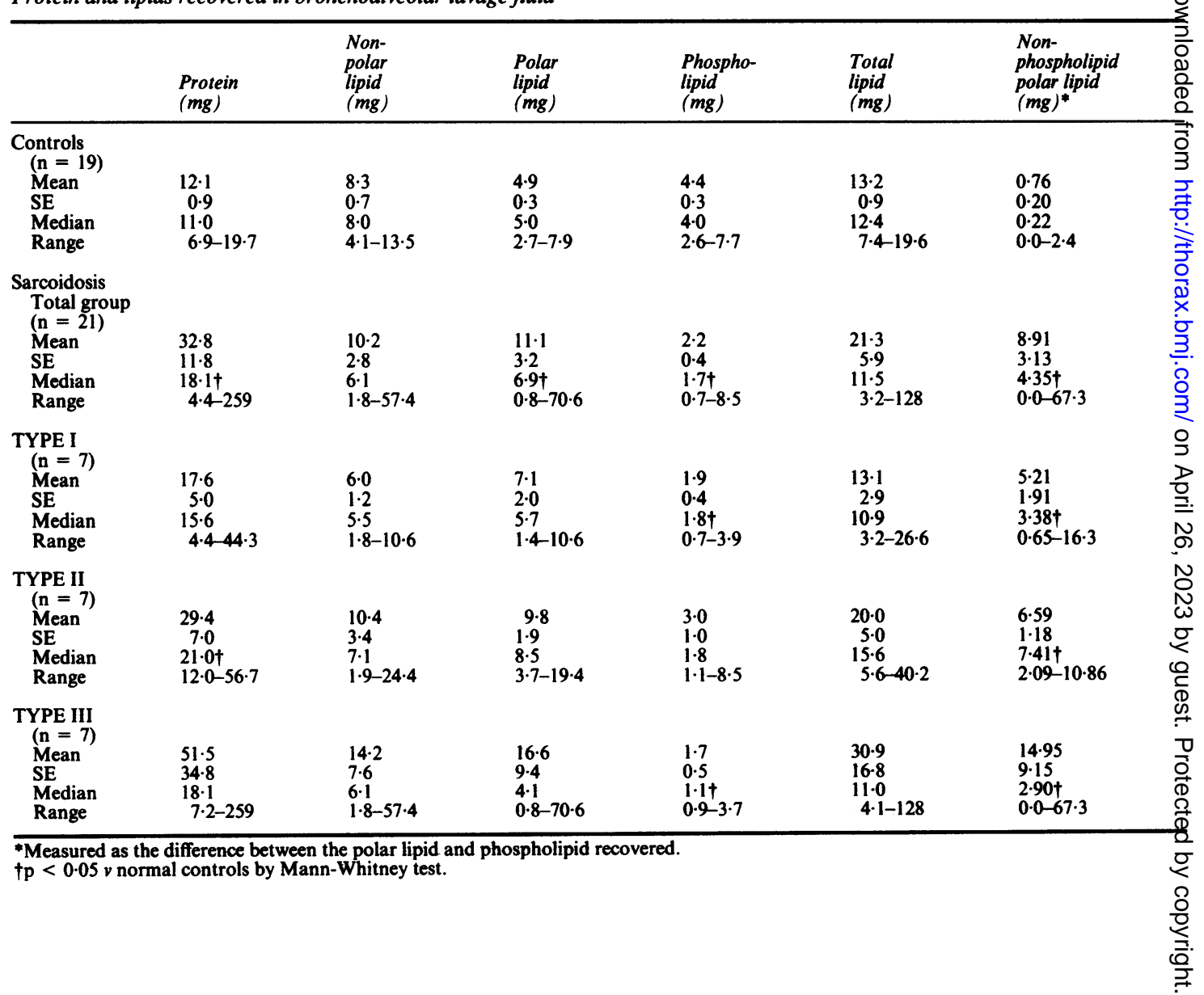



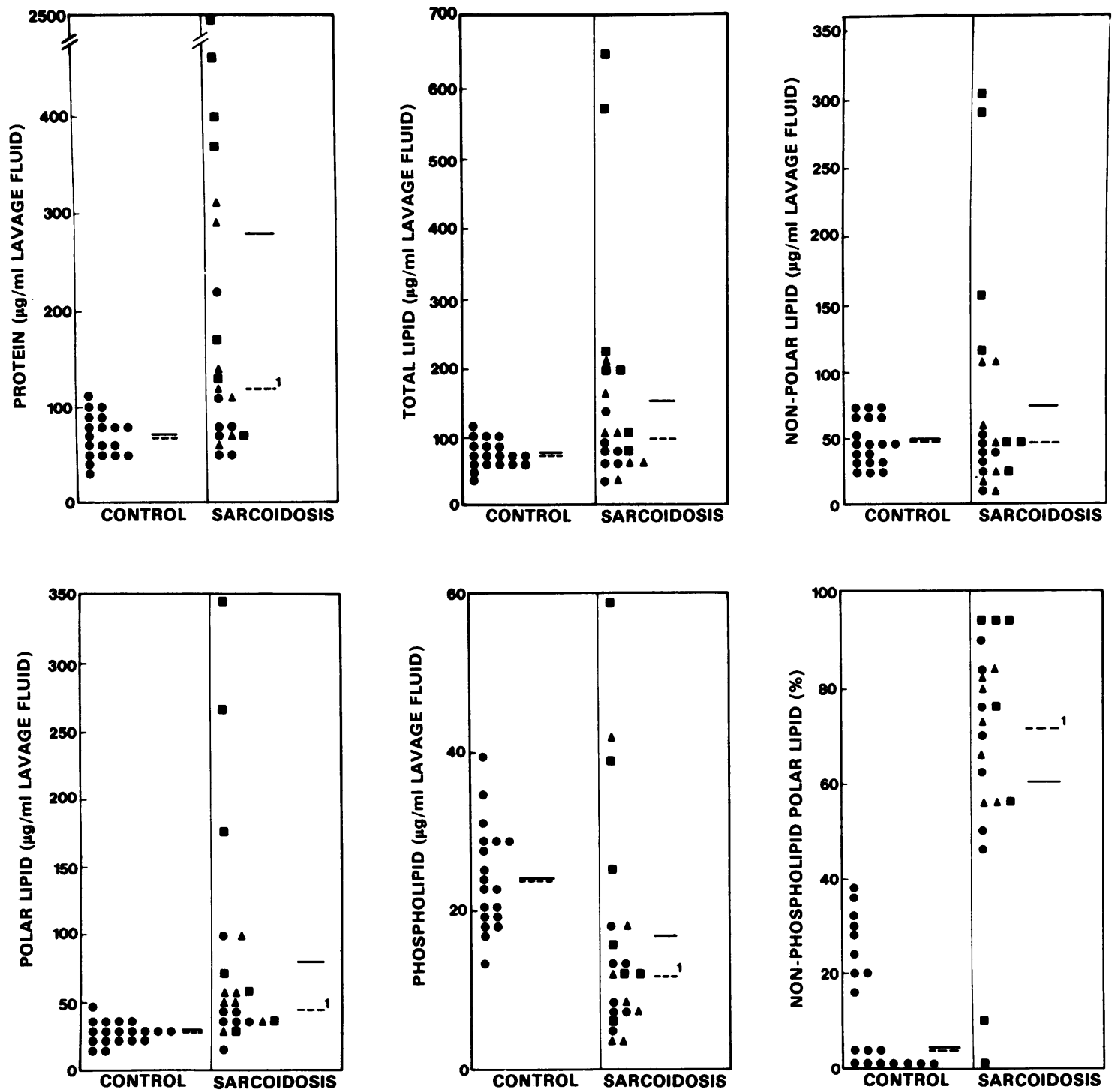

Scattergrams showing measurements of lipids and proteins in bronchoalveolar lavage fluid. — Mean; - - - median. - Type I sarcoidosis; $\triangle$ type II; 1 type III. 'p < $0.05 \mathrm{v}$ normal controls by Mann-Whitney test.

The amount and concentration of protein recovered were significantly greater in lavage fluid from patients than from normal subjects ( $p<0.05$; table). There was no difference in protein values between sarcoidosis subtypes but trend analysis showed that protein values increased in the order type I < type II < type III (p < 0.05 ). In all cases the predominant protein was albumin, with no change in the range of proteins present, as indicated by polyacrylamide gel electrophoresis in the presence of sodium dodecyl sulphate (not shown).
LIPID ANALYSES

The recoveries and concentrations of total lipid and non-polar lipid for lavage fluid from patients with sarcoidosis were not significantly different from the corresponding values for normal subjects (figure and table). The amount and concentration of phospholipid recovered were significantly less than for volunteers ( $p<0.05$ ), although polar lipid values were nevertheless higher in the sarcoidosis group $(p<0.05)$. The combined polar lipid and phospholipid data indicated substantial increase in the amounts of 
nonphospholipid polar lipid ( $p<0.05$ ).

The predominant non-polar lipids in both the normal subjects and the groups of patients were triglyceride, cholesterol, and cholesterol ester. Median values of percentage of non-polar lipids for triglyceride (control 14\%, sarcoidosis patients 15\%) and cholesterol esters (median values $35 \%$ v $31 \%$ ) were not significantly different for the two groups. There was a significant reduction in median values for cholesterol as percentage of non-polar lipid (control 53\%, sarcoidosis $35 \% ; p<0.05)$ and in terms of the total recovered (control $3.2 \mathrm{mg}$, sarcoidosis $1.5 \mathrm{mg}$; p < 0.05).

The fatty acids 1,2-diglyceride and 1,3-diglyceride ( $<10 \%$ of total non-polar lipid) were recovered in smaller amounts and were not detected in lavage fluid from several normal subjects and patients.

The phospholipid chromatograms for samples from the two study groups also showed no differences. Two surfactant lipids predominated, phosphatidylcholine (median values as percentage of total phospholipid: controls $85 \%$, patients $83 \%$ ) and phosphatidylglycerol (median values: $12 \% \quad v \quad 12 \%$ ). Diphosphatidylglycerol, phosphatidylethanolamine, lysophosphatidylcholine, and phosphatidylinositol were either not present or present in small amounts $(0-5 \%$ of total phospholipid). About half of the phospholipid was saturated in both groups.

The only difference between sarcoidosis types was that observed between total lipid concentrations in samples from patients with type I and those from patients with type III radiographs (I < III: $p<0.05$ ). Trend analyses, however, showed that polar lipid, non-polar lipid, and hence total lipid concentrations increased in the order type I < type II $<$ type III $(\mathrm{p}<$ $0.05)$.

\section{CORRELATIONS}

There were few strong correlations between measurements for either study group. Accepting $r>$ 0.5 and $p<0.05$ as indicating statistical significance, we found significant correlations in normal subjects between total number of cells recovered and protein concentration $(r=0.58 ; p<0.005)$ and between cell concentration and protein concentration $(r=0.59$; $\mathrm{p}<0.004)$. These correlations did not hold for the patients with sarcoidosis, though in this group there was a positive correlation between percentage of lymphocytes and phospholipid concentration $(r=$ $0.55 ; p<0.005)$. There were no significant correlations for either group between lavage fluid biochemical measurements and age, sex, or smoking history.

We were concerned that certain of the differences we observed might be due to the volumes of our lavage fluids recovered, even though these did not differ significantly between the two groups. There were $\stackrel{?}{?}$ however, no strong correlations between volume? recovered and any of the biochemical variables $\left(r<\frac{\bar{D}}{\bar{C}}\right.$ 0.5 and $p>0.05$ ). Furthermore, we observed the same $\frac{\vec{\sigma}}{\widehat{C}}$ significant differences, and no new ones, when the 0 comparisons were restricted to the 16 patients who had is undergone complete lavage.

\section{DISCRIMINANT ANALYSIS}

The discriminant function we obtained consisted of the following variables, given with their standardised $x$ coefficients and in decreasing order of their contri- $\vec{t}$ bution to the discriminant function: non-phospholipid $\vec{N}$ polar lipid (1.053), protein $(0.852)$, non-polar lipidio $(-0 \cdot 241)$. This function correctly classified all 40 of $\tilde{\sigma}$ the subjects in our study, high scores identifying patients with sarcoidosis. It also correctly classified all $\rightarrow$ but one control subject when applied to a new group of nine normal subjects and patients (not shown), indicating that the variables selected and the equation 3 used are not artefacts of the particular data set from@ which they were derived.

\section{Discussion}

The goal of our studies was to determine what changes occur, if any, in lavage surfactant lipids in sarcoidosis. Several distinctive abnormalities were observed, reflecting a different pattern of change than that 2 found in non-granulomatous interstitial disease. $\overrightarrow{\overrightarrow{0}}$ These abnormalities enabled us to distinguish patients 3 with sarcoidosis from volunteers with a high degree of accuracy and were related to alterations in chest radiographs.

Our finding of reduced phospholipid concentrations in our patients with sarcoidosis confirms an earlier $\times$ report by Baughman et al, ${ }^{21}$ who studied 13 patients $\frac{3}{3}$ but did not provide data regarding the radiographic. type of sarcoidosis. They also reported a change $\frac{\mathrm{O}}{3}$ in the fatty acid composition of recovered phosphatidylcholine. Most of the phospholipid present in all $\mathrm{O}$ samples was saturated phosphatidylcholine and $\$$ phosphatidylglycerol. These phospholipids are major products of pulmonary type II epithelial cells. ${ }^{5}$ Thus $N$ our results suggest a reduced ability of these cells to synthesise or export (or both) surfactant constituents in sarcoidosis. Alternatively, there could be alterations ${ }^{\mathrm{C}}$ in epithelial cell uptake mechanisms or other aspects? of pulmonary clearance of these substances. ${ }^{22-24}$ The degree to which these changes in phospholipids, together with the fall in cholesterol, might affect ${ }^{+}$ physiological functions of the lung in terms of altered $\frac{0}{T}$ surfactant activity remains to be further explored.

Our finding of increased recovery of protein may $\frac{\odot}{\Phi}$ relate to the lipid abnormalities. Our hypothesis is that $\stackrel{\mathbb{2}}{2}$ this is due partly to injury or damage to the alveolar 
capillary membrane, leading to altered flux of serum proteins, ${ }^{25}$ since the vast majority of the protein is albumin, the proportion of which does not appear to be different from control values based on electrophoretic analysis (data not shown in results). Altered local production of immunoglobulins is also a likely contributor. Increased concentrations of proteins and other constituents might also be due to a defect in their clearance from the alveolar surface. Finally, protein could accumulate at the alveolar surface as a result of bronchiolar or airway damage. In any case, the lack of a correlation between protein and cell recovery for the patients with sarcoidosis, in contrast to that found in lavage fluid from normal subjects, suggests that whatever mechanism is responsible for the increase in lavage protein does not cause a similar change in the number of cells recovered in the lavage fluid.

Extracts containing pulmonary surfactant phospholipids suppress the in vitro proliferative response of blood lymphocytes to mitogenic, antigenic, or allogenic stimulation. ${ }^{26}$ This may explain why lymphocytes recovered from the lungs of healthy individuals show less in vitro response to such stimuli than do peripheral blood lymphocytes. ${ }^{27}$ Thus reduced amounts of lavage phospholipids, such as we found in fluid from our patients with sarcoidosis, might contribute to a failure to regulate lymphocyte function in this disease. Noteworthy in this respect is the correlation we observed between lymphocytes and phospholipid recovered from patients with sarcoidosis.

The pattern of change in surfactant constituents that we observed for patients with sarcoidosis is quite different from that observed in non-granulomatous interstitial disease. Hallman et al ${ }^{6}$ reported a reduction in the proportion of phosphatidylglycerol relative to phosphatidylcholine in lavage fluid from patients with the adult respiratory distress syndrome. Our own preliminary studies of idiopathic pulmonary fibrosis documented by biopsy have produced similar data (mean PG/PC $=0.05$ (SE 0.01), median $=0.04$, $\mathrm{n}=11 ; \mathrm{p}<0.05){ }^{7}$ Similar changes occur in acute lung injury induced by $N$-nitroso- $N$-methylurethane in dogs, ${ }^{11}$ and by oxygen ${ }^{89}$ and silica ${ }^{10}$ in rats. These interesting changes may reflect a return of type II cell lipid metabolism to a pattern seen during fetal and postnatal lung development. ${ }^{5}$

Interpretation of the meaning of increased concentrations of non-phospholipid polar lipid in lavage samples from patients with sarcoidosis awaits further characterisation. The fraction appears not to include the substantial amounts of proteolipids and glycolipids shown by protein and carbohydrate analyses of the polar lipid fraction (data not shown). Partial phospholipid hydrolysis products also appear not to be major constituents, to judge by a series of chromatographic analyses (data not shown). It also must be borne in mind that this is a calculated value based on two measurements, of polar lipid and of lipid phosphorus, and thus could include a relatively large composite error. In a new series of lavage studies in patients with sarcoidosis patients we have found that increased concentrations of this peculiar lipid fraction correlate with decreased lavage cell viability as well as with two apparent markers of the disease, increased serum IgG and increased serum caeruloplasmin concentrations ${ }^{28}$ (unpublished observations).

Comparison of biochemical markers with different types of radiographic change in sarcoidosis proved rewarding. Patients with type I radiographs showed a striking decrease in lipid phosphorus and increase in non-phospholipid polar lipid in lavage fluid. Nonphospholipid polar lipid recovery was increased in patients with type II and type III changes but phospholipid recovery decreased only for the latter. The relationship between these changes and radiographic evidence of parenchymal disease remains unclear, given that patients with type I disease may have biopsy evidence of parenchymal disease. ${ }^{29}$ The trends we found, however, lend support to the notion that the sequence of types reflects disease progression even though individual patients do not necessarily advance through such a sequence. ${ }^{30}$ Such a conclusion will become more justified if supported by longitudinal studies of individual patients. In preliminary studies we have found that abnormal values for protein and polar lipid have returned towards normal in three patients as their clinical condition underwent remission, while values remained abnormal or became more so in one patient whose condition worsened.

Standard univariate analyses do not take account of interrelationships between variables that might help to distinguish groups and aid understanding of pathogenesis. Discriminant analysis can provide such information by identifying subsets of variables that account for most of the differences between groups. ${ }^{20}$

Our discriminant analysis indicates that three of the biochemical markers (non-phospholipid polar lipid, protein, and non-polar lipid) collectively have the ability to distinguish patients with sarcoidosis from normal subjects. Sarcoidosis patients were characterised by an increased proportion of non-phospholipid polar lipids or an increased protein concentration, or both, not accompanied by high concentrations of non-polar lipids. It was the proportion of polar lipids that were non-phospholipid, rather than the actual concentration, which distinguished the patients with sarcoidosis from the normal subjects. Further work, based on larger numbers, is necessary to refine the discriminant function and to determine its value in the clinical and research areas. In particular, it will be important to determine whether the biochemical markers can also differentiate different groups of patients from each other, as our own preliminary analyses suggest. ${ }^{7}$ In conclusion, our hypothesis is that measurement of change in cell function related to 
protein permeability and lipid metabolism may be important in distinguishing granulomatous diseases such as sarcoidosis from other interstitial lung disorders. Many questions regarding the meaning of altered concentrations of surfactant constituents of lavage fluid in interstitial lung disease remain unresolved. Nonetheless, we believe that a fuller definition of the distinctive changes we and others have observed will lead to a better understanding of the pathogenesis of these diseases.

We thank Dr Jason Kelley for many helpful discussions, and Ms Julee Bode, Ms Ruth Furness, Ms Margaret Smith, and Ms Janice Gregoire for excellent secretarial assistance with the manuscript. Finally, we thank the referees for their valuable comments. This work was supported by grants from the National Heart, Lung, and Blood Institute-PHS HL 14212 (SCOR) and PHS RR-109 (general chemical research centers program).

\section{References}

1 Reynolds HY, Newball HH. Fluid and cellular milieu of the human respiratory tract. In: Kirkpatrick $\mathrm{CH}$, Reynolds HY, eds. Immunologic and infectious reactions in the lung. New York: Marcel Dekker, 1976:3-27.

2 Davis GS, Landis JNN, Brody AR, Graham WGB, Craighead JE, Green GM. Characteristics of diffuse lung disease reflected by pulmonary lavage. Am Rev Respir Dis 1975;111:933-4.

3 Haslam PL, Turton CWG, Heard B, et al. Bronchoalveolar lavage in pulmonary fibrosis: comparison of cells obtained with lung biopsy and clinical features. Thorax 1980;35:9-18.

4 Hunninghake GW, Kawanami O, Ferrans VJ, Young RC Jr, Roberts WC, Crystal RG. Characterization of the inflammatory and immune effects of cells in the lung parenchyma of patients with interstitial lung disease. Am Rev Respir Dis 1981;123:407-12.

5 Rooney SA. The surfactant system and lung phospholipid biochemistry. Am Rev Respir Dis 1985;131:439-60.

6 Hallman M, Spragg R, Harrell JH, Moser KM, Gluck L. Evidence of lung surfactant abnormality in respiratory failure. Study of bronchoalveolar lavage phospholipids, surface activity, phospholipase activity and plasma myoinositol. J Clin Invest 1982;70:673-83.

7 Low RB, Davis GS, Bell DY, Giancola MS, Vacek PM. Discrimination between volunteer, sarcoidosis and idiopathic pulmonary fibrosis patients by lavage biochemical analyses [abstract]. Am Rev Respir Dis 1984;129:22A.

8 Baker G, Duck-Chong C, Cleland K, Berend N. The surfactant system as an intrinsic monitor of acute interstitial lung disease. Chest 1986;89:126-7.

9 Crapo JD, Barry BE, Foscue HA, Shelburne J. Structural and biochemical changes in rat lungs occurring during exposures to lethal and adaptive doses of oxygen. Am Rev Respir Dis 1980;122:123-43.

10 Dethloff LA, Gilmore LB, Brody AR, Hook GER. Induction of intra- and extra-cellular phospholipids in the lungs of rats exposed to silica. Biochem $J 1986 \stackrel{5}{+}$ 233:111-8.

11 Liau DF, Barrett CR, Bell ALL, Cernansky G, Ryan ST Diphosphatidylglycerol in acute experimental alveolaf injury in the dog. J Lipid Res 1984;25:678-83.

12 Sharma OP. Sarcoidosis: a clinical approach, Springfiel $\phi_{3}$ Illinois: Chas C Thomas, 1976:30-64.

13 Low RB, Davis GS, Giancola MS. Biochemical analys of bronchoalveolar lavage fluids of healthy human volunteer smokers and non-smokers. Am Rev Respy Dis 1978;118:863-75.

14 Marinetti GV. Lipid chromatographic analysis. New⿳亠丷冖 York: Marcel Dekker, 1976.

15 Freeman CP, West D. Complete separation of lipid classes on a single thin-layer plate. J Lipid Res 1960 7:324-7.

16 Wuthier RE. Lipid composition of isolated epiphysea cartilage cells, membranes and matrix vesicles. Biochem Biophys Acta 1975;409:128-43.

17 Mason RJ, Nellenbogen J, Clements JA. Isolatio of disaturated phosphatidylcholine with osmiug tetroxide. J Lipid Res 1976;17:281-4.

18 Dixon WJ, Massey FJ Jr. Introduction to statistic analysis. New York: McGraw Hill, 1969:391-2.

19 Peto R, Pike MC, Armitage P, et al. Design and analysis of randomized clinical trials requiring prolonged observations of each patient. Br J Cancer 1977;35:1-39.

20 Morrison DF. Multivariate statistical methods. New York: McGraw-Hill, 1967:131-3.

21 Baughman RP, Strohofer S, Dohn M. Decreased pho phatidylcholine in the lung fluid of patients wifh sarcoidosis. Lipids 1985;20:496-9.

22 Chander A, Claypool WD, Strauss JF, Fisher AB Uptake of liposomoal phosphatidylcholine by granula pneumocytes in primary culture. Am J Physiol 1983.; 245:397-404.

23 Claypool WD, Wang DL, Chander A, Fisher AB. Aiti ethanol/ether soluble apoprotein from rat lung surfact tant augments liposome uptake by isolated granulaig pneumocytes. J Clin Invest 1984;74:677-84.

24 Jacobs H, Jobe A, Ikegani M, Miller D, Jones Reutilization of phosphatidylcholine analogues by the pulmonary surfactant system. Biochim Biophys Ac? 1984;793:300-9.

25 Staub NC. Pulmonary edema. Physiol Rev 1974;5 678-811.

26 Anfield MJ, Kaltreider HB, Benson BJ, Coldwell HE Immunosuppressive activity of canine pulmonary suf face active material. J Immunol 1980;122:1062-6. N

27 Anfield MJ, Kaltreider HB, Benson BJ, Shalaby MR, Canine surface active material and pulmona lymphocyte function. Studies with mixed-lymphocyte culture. Exp Lung Res 1980;1:3-11.

28 Bell DY, Spock A, Piantadosi CA, Johnson SM. Serum IgG and ceruloplasmin concentrations as indicators high intensity alveolitis in the lung in sarcoidos $s$ [abstract]. Am Rev Respir Dis 1983;127:89A.

29 Koerner SK, Sakowitz AJ, Appleman RI, Beck NI6 Schoenbaum SW. Transbronchial lung biopsy for diagnosis of sarcoidosis. N Engl J Med 1975;298 268-70.

30 Siltzbach LE, ed. Seventh international conference on sarcoidosis and other granulomatous disorder. Ann Nल Acad Sci 1976;278:1-751. 